\title{
Selection of an optimal muscle set for a 16-channel standing neuroprosthesis using a human musculoskeletal model
}

\author{
Benjamin P. Heilman, MS; Musa L. Audu, PhD; ${ }^{*}$ Robert F. Kirsch, PhD; Ronald J. Triolo, PhD \\ Department of Biomedical Engineering and Orthopedics, Case Western Reserve University, Cleveland, OH; Func- \\ tional Electrical Stimulation Center of Excellence, Louis Stokes Cleveland Department of Veterans Affairs Medical \\ Center, Cleveland, $\mathrm{OH}$
}

\begin{abstract}
This study proposes an optimal set of lower-limb muscles to be stimulated electrically with a 16-channel neuroprosthesis that will allow persons with paraplegia caused by spinal cord injury to stand and shift postures smoothly, thus minimizing muscle fatigue and facilitating performance of activities of daily living. We used a three-dimensional (3-D) 15 degree-of-freedom musculoskeletal model of the human lower limbs to assess different muscle combinations that would maintain specific standing postures while minimizing the overall metabolic energy consumed. We initially selected the postures by discretizing the joint-angle space over the ranges of the knee, hip, and ankle angles and then refined the postures by relating the lower-limb joint angles to the center of mass (COM) of the musculoskeletal model to generate smooth transitions between desired postures. We found a set of four 3-D second-order polynomials adequate for obtaining the best fit between the joint angles and the COM components. The results showed that adding the gluteus medius and the adductor magnus to balance nonsagittal movements at the hip and adding several different combinations of ankle muscles should allow users to shift postures over $75 \%$ of the forward-backward range that nondisabled individuals use during typical activities. The simplest complete ankle-muscle set only requires the soleus and the tibialis anterior, and the medial and lateral gastrocnemii could be added for additional plantar flexion. Alternatively, if the ankle is consistently being inverted, the peroneus muscles could be added.
\end{abstract}

Key words: bipedal balance, functional electrical stimulation, lower-limb musculature, muscle selection, musculoskeletal model, neuroprostheses, posture shifting, rehabilitation engineering, spinal cord injury, standing balance, unassisted standing.

\section{INTRODUCTION}

Approximately 200,000 people with spinal cord injuries (SCIs) live in the United States [1]. About 20 percent of these injuries resulted in complete paraplegia, described as paralysis of all muscles below the level of injury to include the muscles of the trunk and lower limbs. The significant functional losses from paralysis, such as the inability to stand or walk, often severely limit the independence, vocational opportunities, and social integration of this group of people [2]. Electrical stimulation of paralyzed muscles has enabled some of these individuals to stand and walk. Several research groups have been successful in developing lower-limb standing systems for persons with paraplegia [3-5]. Our group at the Case Western Reserve University/Cleveland Department of Veterans Affairs (VA) Medical Center (CWRU/VAMC)

\footnotetext{
Abbreviations: $\mathrm{AFO}=$ ankle-foot orthosis, $\mathrm{BOS}=$ base of support, $\mathrm{COM}=$ center of mass, CWRU/VAMC = Case Western Reserve University/Cleveland Department of Veterans Affairs Medical Center, $d f=$ degrees of freedom, FES = functional electrical stimulation, SCI = spinal cord injury, SQP = Sequential Quadratic Programming, VA = Department of Veterans Affairs.

*Address all correspondence to Musa L. Audu, Motion Study Laboratory $151 \mathrm{~A}(\mathrm{~W})$, Cleveland VA Medical Center, Cleveland, OH 44106; 216-791-3800, ext. 3821; fax: 216231-3433. Email: musa.audu@case.edu

DOI: 10.1682/JRRD.2005.04.0072
} 
developed an 8-channel functional electrical stimulation (FES) implantable system that allows persons with low cervical-level SCI to stand and transfer with minimal assistance [6-7]. Along with stimulation of knee, hip, and trunk extensors, the ankle is locked with an ankle-foot orthosis (AFO), and the user relies on upper-limb support and a walker or other assistive device to remain in a standing position. Users have been able to stand with minimal upper-limb assistance, and many have been able to free one hand to perform functional activities [8].

Exercise and weight-bearing with FES are reported to improve cardiovascular fitness and increase bone density without adverse effects on the insensate joints [9]. Furthermore, exercise and weight-bearing may reduce the risk of developing pressure ulcers by improving tissue oxygen levels, increasing muscle bulk, and altering seated pressure distribution [10].

Although the benefits of the FES standing systems are numerous, the potential for improvement still exists. Standing-system users are unable to initiate anticipatory postural adjustments to perform activities such as picking up a book or resist undesirable posture changes due to fatigue or external disturbances except through the use of the upper limbs. In addition, the CWRU/VAMC standing system relies on an AFO to lock the ankle, leaving the ankle angle uncontrolled. Because of its distal location, the ankle has quite a large effect on postural stability of the user while standing, so controlling the ankle would potentially provide a more flexible control strategy [11].

A 16-channel stimulation system that enhances standing performance relative to the 8-channel CWRU/ VAMC system would help achieve this flexible anklecontrol strategy. Such a system is currently being developed in our laboratory. Before it can be optimally used, we must clearly determine which additional muscles would need to be stimulated to get the ideal outcomes. Since obtaining this information by direct experimentation with live subjects would be prohibitively difficult, we addressed the issue in a simulation study using a three-dimensional musculoskeletal model. Specifically, we conducted inverse computations to suggest 16 muscle sets that would allow users of the FES system to adjust their postures with a user-controlled device over a functionally relevant range while standing. This approach allowed us to evaluate the feasibility of providing enhanced standing function to individuals with SCI without requiring human subjects to undergo invasive and tedious experimental testing.

\section{METHODS}

\section{Musculoskeletal Model}

We used a bipedal standing model in this study [12]. It consisted of a system of 10 rigid bodies made up of the thorax (head, arms, and trunk) and the pelvis. It also had the talus, foot (calcaneus, mid-foot, metatarsals, and toes), tibia/fibula, and femur (all for both right and left lower limbs). In total, the model contained 15 degrees of freedom $(d f): 3$ at the lumbosacral joint and, bilaterally, 2 at the ankle, 1 at the knee, and 3 at the hip. The kinematics of the lumbosacral joint were included to allow for different postures, but the model lacked muscles spanning the lumbosacral joint so no muscle activations were computed for this joint. The bilateral erector spinae are included in the set of muscles used in the 8-channel FES system. Thus, the required lumbosacral moments were available and included in the simulations. However, we did not explicitly compute the corresponding muscle activations. With the two feet planted on the ground, the bipedal standing-model system defines a closed kinematic chain. Because of the presence of algebraic constraint equations in addition to the differential dynamic equations, the solution of the dynamic equations for such a system is complicated. The closed kinematic chain introduces a redundancy in the system that is difficult to resolve with the laws of mechanics alone. We dealt with the closed kinematic chain consisting of the two lower limbs and the ground by fixing one foot to the ground while supporting the other foot with a vertical reaction force. In this way, the kinematic chain is opened, allowing for a simpler system of dynamic equations and an easier solution of the joint moments in the system [12].

For this study, the complete set of muscles readily accessible via implanted electrodes in FES systems included in the model are summarized in Table $\mathbf{1}$ [13].

\section{Model Assumptions}

To balance complexity, computation time, and accuracy, we assumed the following:

1. The feet were shoulder width apart and placed symmetrically about the sagittal plane. Although alternative positions of the feet could be examined using the model, we examined only one set of foot locations.

2. The knee, ankle, and hip angles of the left leg were set equal to those of the right leg throughout this study, creating symmetry about the midsagittal axis 
Table 1.

Modeled muscles and their actions. Muscles included in computation and their corresponding actions.

\begin{tabular}{|c|c|}
\hline Muscle & Action \\
\hline Gluteus Maximus* $^{*}$ & Hip: extension, lateral rotation \\
\hline Gluteus Medius (anterior) & $\begin{array}{l}\text { Hip: abduction, medial rotation, } \\
\text { slight flexion }\end{array}$ \\
\hline Gluteus Medius (posterior) & $\begin{array}{l}\text { Hip: abduction, lateral rotation, } \\
\text { slight extension }\end{array}$ \\
\hline Adductor Magnus (anterior) & Hip: adduction, slight flexion \\
\hline \multirow[t]{2}{*}{ Biceps Femoris Long Head } & Knee: flexion \\
\hline & $\begin{array}{l}\text { Hip: extension, adduction, } \\
\text { lateral rotation }\end{array}$ \\
\hline \multirow[t]{2}{*}{ Semimembranosus ${ }^{*}$} & Knee: flexion \\
\hline & $\begin{array}{l}\text { Hip: extension, adduction, } \\
\text { medial rotation }\end{array}$ \\
\hline \multirow[t]{2}{*}{ Rectus Femoris } & Knee: extension \\
\hline & Hip: flexion \\
\hline Vastus Lateralis ${ }^{*}$ & Knee: extension \\
\hline Tibialis Anterior & Ankle: dorsiflexion, inversion \\
\hline Peroneus Brevis & Ankle: eversion, plantar flexion \\
\hline Peroneus Longus & Ankle: eversion, plantar flexion \\
\hline Peroneus Tertius & Ankle: eversion, dorsiflexion \\
\hline Soleus & Ankle: plantar flexion, inversion \\
\hline \multirow[t]{2}{*}{ Medial Gastrocnemius } & Ankle: plantar flexion, inversion \\
\hline & Knee: flexion \\
\hline \multirow[t]{2}{*}{ Lateral Gastrocnemius } & Ankle: plantar flexion, inversion \\
\hline & Knee: flexion \\
\hline
\end{tabular}

because we only examined postures extending through the sagittal plane.

3. The masses of the head, arms, and trunk of the body were lumped and positioned dependent on the body posture. Additionally, the user was assumed to have good upper-body control achieved through a brace or similar device.

4. The friction between the bottom of the feet and the ground surface was sufficient to prevent slipping.

5. A static muscle model was assumed adequate for representing quiet standing [14]. Because the velocity was zero, the muscle force was computed as the product of the muscle activation and the maximum isometric muscle force, dependent on the length-tension property of the muscle. The effects of muscle fatigue were excluded from this study.
6. The specific muscle and geometrical parameters used in the model were based on cadaver measurements. The model height and mass were $1.76 \mathrm{~m}$ and $78.6 \mathrm{~kg}$, respectively. The model contained anatomically accurate muscle representations, with specified origins and insertions for 46 muscles (23 bilaterally) [15-17]. Including all 46 muscles or just the subset used by a particular FES system was possible. Because of the large ranges of the muscle origins of the gluteus maximus, the gluteus medius, and the adductor magnus, each muscle was represented by three muscle elements.

7. Maximum stimulated muscle forces in an individual with chronic SCI who has completed an FES-mediated exercise program were assumed to be 50 percent of the values of a typical nondisabled individual.

8. Linear recruitment properties of electrically activated muscle were assumed. The specific recruitment properties of different muscles are often quite variable but can be considered based on experimental measurements.

9. Muscle selectivity was assumed perfect, i.e., a single stimulating electrode recruited only the intended muscle without spillover to the other muscles [18].

10. Multiple muscles can in some cases be purposely activated with a single stimulation channel. This can be accomplished in several ways: branching an electrode to stimulate two muscles [19], placing a single electrode near the motor points of adjacent muscles [20], or stimulating a nerve proximal to the point where it branches to several muscles simultaneously [3].

11. Postures that required lumbosacral moments greater than $90 \mathrm{~N} \cdot \mathrm{m}$ cannot be maintained by individuals with thoracic SCIs. For postures requiring less than $90 \mathrm{~N} \cdot \mathrm{m}$ at the lumbosacral joint, we assumed that a combination of retained volitional muscle control and electrical stimulation of the erector spinae could produce adequate moments to stabilize the lumbosacral joint. We determined the $90 \mathrm{~N} \bullet \mathrm{m}$ threshold by using the nominal nondisabled maximum trunk moment of $250 \mathrm{~N} \cdot \mathrm{m}$ in a standing position [21], accounting for the effects of paralysis (50\% of nondisabled), and including a 25 percent safety factor.

12. The resistance of passive tissues (ligaments, joint capsule, connective tissues within muscle) to lowerlimb joint rotation following thoracic SCI was assumed to be as described by Amankwah et al. [22]. 
13. We solved the redundant force-sharing problem by minimizing the sum-of-squared stress across all of the activated muscles. This strategy distributed the load across the whole set of muscles, presumably delaying the onset of fatigue [23].

14. A 16-channel stimulation system was available to activate paralyzed muscles of the lower limbs, pelvis, and lower trunk.

\section{Determining Feasible Postures}

We selected an initial set of postures by equally dividing the range of each joint angle (ankle, knee, hip, and lumbosacral) in the sagittal plane into 10 regions and examining all possible combinations across these divisions. We eliminated postures that resulted in the center of mass (COM) being located outside of the base of support (BOS) and those with a vertical COM value below $0.8 \mathrm{~m}$ (maximum vertical COM is $0.98 \mathrm{~m}$ ) to avoid statically unstable postures and excessively crouched positions, respectively.

For the remaining postures, we performed inverse dynamic calculations to determine the joint moments required to maintain them. Thereafter, we used the Sequential Quadratic Programming (SQP) optimization algorithm (e04ucf.m, NAG/MATLAB Optimization Toolbox, The MathWorks, Natick, Massachusetts) to determine the muscle activations that would generate the same moments. The objective function for the optimization was the sum of squares of the muscle stresses for that set of muscles. Running the optimization program with all 46 lower-limb muscles in the model indicated the maximum performance possible and thus eliminated all postures that would be impossible with any reduced muscle set. Feasible postures then were those that could be supported statically with the set of muscles used in the particular run.

The feasible postures reduced the joint-angle ranges. These new joint-angle ranges were then redivided evenly into 10 subdivisions. Next, we used these subdivisions to generate entirely new postures with the new combinations of joint angles. Some of these combinations would lead to postures in which the COM falls outside the restricted boundary. The postures falling outside of the COM restrictions were eliminated, and the muscle activation optimization program with the complete muscle set was applied to the resulting postures. The maximum possible range of motion of the COM was thus taken as the set of postures arising from this last step.

\section{Functional Stability Region}

The final set of postures determined in the preceding section represent body positions that produce anteroposterior positions of the COM within the absolute limits of the BOS defined by the boundary of the two feet. Others have shown that the COM is more typically maintained within a smaller range during functional tasks [24]. This functional stability region, illustrated in Figure 1, spans 58 percent of the BOS, excluding the 19 percent closest to the heel and the 23 percent closest to the toes. Although the posture sets were limited by the BOS, the results will be presented relative to both the BOS and the functional stability region.

\section{Hip Muscle Selection}

The existing 8-channel CWRU/VAMC FES standing system stimulates two hip extensor muscles (gluteus maximus and semimembranosus) that are adequate to provide upright standing for reasonable periods $[6,8]$. However, stronger hip extension moments should provide longer standing times and better performance for heavier and taller individuals. Additional hip muscles could also be

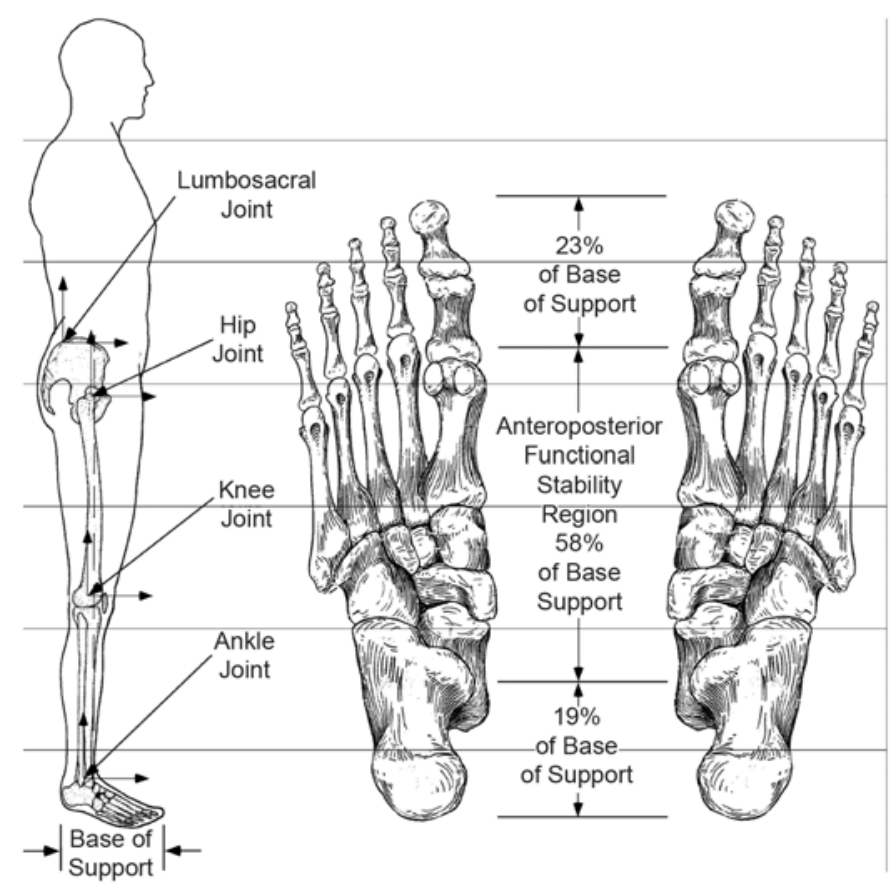

Figure 1.

Functional stability region. A diagram of sagittal plane range of functional stability region compared with base of support is shown for human skeletal system. Positions of 4 degrees of freedom in sagittal plane are also labeled. 
used to balance hip moments in abduction/adduction and in the internal/external rotation produced by the primary hip extensors and potentially allow coronal plane posture shifts. We targeted several different sets of hip muscles because of their ability to produce the largest moments in each of these $d f$ in the neutral position of the hip. The objective is to select two additional muscles bilaterally from the five hip muscles listed in Table 2 . These include the anterior portion of the gluteus medius because it generates the largest moment in both medial rotation and hip abduction. Others are rectus femoris for flexion, long head of biceps femoris for extension, and anterior portion of adductor magnus for adduction, and the posterior portion of gluteus medius for internal rotation. In addition, all computations to select hip muscles included the complete set of available ankle muscles so that the postures maintained by the hip muscles would not be limited by the ankle.

\section{Knee Muscle Selection}

Our main interest in this study is in the selection of appropriate hip and ankle muscles. The existing 8-channel system stimulates the vastus lateralis, the largest and strongest uniarticular head of the quadriceps, to extend the knee joint for standing without flexing the hip. These muscles would also be used in the 16-channel FES system. Any residual joint moments generated by two-joint muscles that cross both the knee and the ankle joints in our selection were considered when computing the needed forces in the quadriceps muscles.

\section{Ankle Muscle Selection}

Ankle muscle selection focused on plantar flexion and dorsiflexion capabilities because previous work has demonstrated the importance of these actions for FES standing [11]. The current 8-channel FES system does not stimulate any ankle muscles. The addition of a plantar flexor muscle and a dorsiflexor muscle bilaterally should provide the ability to shift in the anteroposterior direction, provide stabilizing cocontraction, and reduce reliance on orthoses and the upper limbs for support. An initial analysis found the 15 ankle-muscle combinations presented in Table 3 to be most successful in supporting the postures, and hence, they were further examined in this study. For these computations, the posture set was limited to the successful positions achieved in the hip-muscle trials.

One can most typically use a single stimulation channel to selectively activate a single muscle, but one can also activate two or more muscles by stimulating a peripheral nerve [3] or by placing the electrode near the motor points of adjacent muscles $[13,18]$. In the case of directly stimulating a peripheral nerve, we assumed equal activation (and hence equal percentage of peak force) of all muscles innervated by the nerve. If one attempts to activate two different muscles by placing the electrode between their motor points, however, the relative activation will depend upon the proximity of the electrode to the nerve branches in each muscle. For modeling purposes, we calculated an activation ratio for the dorsiflexor muscles by balancing the subtalar moment [20] at a single standing posture in the middle of the standing range and then using this ratio in all computations involving that "electrode." Plantar flexion produced by the combined actions of the medial and lateral gastrocnemii relied primarily on the medial gastrocnemius with coactivation of the lateral gastrocnemius. Initially, simulations were run for a range of ankle angles that allowed unrestricted activations of both the medial and lateral gastrocnemii and all five of the different dorsiflexor combinations. Using the results of these simulations, we determined five medial and lateral gastrocnemii activations by the slope of a line fitted between the activations of these two muscles, yielding balanced plantar flexion. In each case, the lateral-medial gastrocnemius coactivation ratio was approximately 1:6.

Table 2.

Hip muscle combinations. Four hip muscle combinations are examined. Muscles included in each combination are shaded and include letter "I." Muscles not included in combination are indicated by a dash (-).

\begin{tabular}{lccccl}
\hline \multicolumn{1}{c}{ Muscle } & \multicolumn{2}{c}{ Combinations } & \multicolumn{2}{c}{ Comment } \\
\cline { 2 - 5 } & $\mathbf{1}$ & $\mathbf{2}$ & $\mathbf{3}$ & $\mathbf{4}$ & Strongest abductor and medial rotator \\
\hline Gluteus Medius (Anterior) & I & I & I & I & Strongest hip flexor \\
Rectus Femoris & I & - & - & - & Strongest hip extensor \\
Biceps Femoris Long Head & - & I & - & - & Strongest adductor \\
Adductor Magnus (Anterior) & - & - & I & - & Strongest lateral rotator \\
Gluteus Medius (Posterior) & - & - & - & I & - \\
\hline \hline
\end{tabular}


Table 3.

Two-channel ankle muscle combinations of plantar flexion and dorsiflexion muscles. Muscles targeted for stimulation with functional electrical stimulation are shaded. Muscles not stimulated are indicated by a dash (-). Numbers in cells for stimulated muscles represent ratios of activations between muscles in that channel.

\begin{tabular}{|c|c|c|c|c|c|c|c|}
\hline \multirow{2}{*}{ Trial } & \multicolumn{3}{|c|}{$\begin{array}{c}\text { Channel } 1 \\
\text { Plantar Flexion Muscles }\end{array}$} & \multicolumn{4}{|c|}{$\begin{array}{c}\text { Channel } 2 \\
\text { Dorsiflexion Muscles }\end{array}$} \\
\hline & $\begin{array}{c}\text { Medial } \\
\text { Gastrocnemius }\end{array}$ & $\begin{array}{c}\text { Lateral } \\
\text { Gastrocnemius }\end{array}$ & Soleus & $\begin{array}{c}\text { Tibialis } \\
\text { Anterior }\end{array}$ & $\begin{array}{c}\text { Peroneus } \\
\text { Brevis }\end{array}$ & $\begin{array}{c}\text { Peroneus } \\
\text { Longus }\end{array}$ & $\begin{array}{c}\text { Peroneus } \\
\text { Tertius }\end{array}$ \\
\hline 1 & - & - & 1.00 & 1.00 & - & - & - \\
\hline 2 & - & - & 1.00 & 1.00 & 1.00 & 1.00 & 1.00 \\
\hline 3 & - & - & 1.00 & 1.00 & - & - & 1.00 \\
\hline 4 & - & - & 1.00 & - & 1.00 & 1.00 & - \\
\hline 5 & - & - & 1.00 & 1.00 & 0.24 & 0.24 & 0.24 \\
\hline 6 & 1.00 & 1.00 & 1.00 & 1.00 & - & - & - \\
\hline 7 & 1.00 & 1.00 & 1.00 & 1.00 & 1.00 & 1.00 & 1.00 \\
\hline 8 & 1.00 & 1.00 & 1.00 & 1.00 & - & - & 1.00 \\
\hline 9 & 1.00 & 1.00 & 1.00 & - & 1.00 & 1.00 & - \\
\hline 10 & 1.00 & 1.00 & 1.00 & 1.00 & 0.24 & 0.24 & 0.24 \\
\hline 11 & 1.00 & 0.11 & - & 1.00 & - & - & - \\
\hline 12 & 1.00 & 0.16 & - & 1.00 & 1.00 & 1.00 & 1.00 \\
\hline 13 & 1.00 & 0.08 & - & 1.00 & - & - & 1.00 \\
\hline 14 & 1.00 & 0.17 & - & - & 1.00 & 1.00 & - \\
\hline 15 & 1.00 & 0.15 & - & 1.00 & 0.24 & 0.24 & 0.24 \\
\hline
\end{tabular}

\section{Mapping Center of Mass Variations via Smooth Joint- Angle Trajectories}

Our final step in this study was to develop an algorithm that would allow an FES user to smoothly vary the sagittal COM of their standing posture by also smoothly varying the four joint angles (ankle $[A]$, knee $[K]$, hip $[H]$, and lumbosacral $[L]$ ). We determined a particular mapping between the commanded COM and the joint angles by simultaneously fitting four second-order polynomial surfaces (one for each joint angle) to the successful postures gained from the ankle-muscle selection procedure. The functional forms of these surfaces are defined by

$$
\begin{aligned}
& A_{p}=f\left(a_{1 i}, X_{C}, Y_{C}\right) \\
& K_{p}=f\left(a_{2 i}, X_{C} Y_{C}\right) \\
& H_{p}=f\left(a_{3 i}, X_{C}, X_{C}\right) \\
& L_{p}=f\left(a_{4 i}, X_{C}, Y_{C}\right),
\end{aligned}
$$

where, $A_{p}, K_{p}, H_{p}$, and $L_{p}$ refer to the predicted ankle, knee, hip, and lumbosacral joint angles and $X_{C}, Y_{C}$ are the anterior-posterior and superior-inferior coordinates of the system COM. The fitting coefficients for the surfaces are given by $a_{l i}, \ldots a_{4 i}$. The subscript $i$ in these coefficients increases from 1 to 6 . All 24 coefficients (6 for each surface) were determined by simultaneously computing the predicted anterior-posterior and superior-inferior coordinates of the COM $X_{C}^{p}, Y_{C}^{p}$ dependent on the predicted joint angles $\left(A_{p}, K_{p}, H_{p}\right.$, and $\left.L_{p}\right)$. Thereafter, we minimized the six functions $F(1)$ to $F(6)$ in Equations (5) to (10) using a multiobjective optimization routine (MATLAB Optimization Toolbox, The MathWorks, Natick, Massachusetts) as

$$
\begin{aligned}
& F(1)=\sum\left(X_{C}^{p}-X_{C}\right)^{2} \\
& F(2)=\sum\left(Y_{C}^{p}-Y_{C}\right)^{2} \\
& F(3)=\sum\left(A_{p}-A\right)^{2} \\
& F(4)=\sum\left(K_{p}-K\right)^{2} \\
& F(5)=\sum\left(H_{p}-H\right)^{2} \\
& F(6)=\sum\left(L_{p}-L\right)^{2} .
\end{aligned}
$$


Equations (1) to (4) relate the two COM components (anterior-posterior and inferior-superior) to each of the four joint angles acting in the sagittal plane (ankle plantar flexion/dorsiflexion, knee flexion/extension, hip flexion/ extension, lumbosacral flexion/extension). This routine thus found a nonunique relationship between COM and the joint angles that guaranteed smooth joint angle transitions when the COM was varied across its feasible range. COM-joint angle surfaces were fitted using postures feasible with the best three sets of ankle muscles. A new set of postures dependent on the set of 24 surface coefficients ( $a_{11}$ to $\left.a_{46}\right)$ were taken from the fitted surfaces, and the muscle activation optimization routine was rerun for verification that the chosen muscle sets were able to produce equilibrium across the fitted surfaces.

\section{RESULTS}

\section{Hip Muscle Selection}

We evaluated four different hip muscle combinations using the muscle activation optimization program. The COM limits for each of the hip combinations are presented in Figure 2. The anteroposterior functional stability region for a nondisabled individual is indicated by the gray area, and the darker gray area indicates the nominal COM range of a person standing still [27]. The connected points indicate the boundary of the COM values that could be stabilized by particular sets of added hip muscles. The combination of the anterior portion of the gluteus medius and the long head of the biceps femoris maintained the most postures and the largest vertical excursion of the COM, but all the combinations supported COM locations across the same range of the anteroposterior functional stability range. Because the different muscle combinations had similar functional attributes, we selected one particular set for all subsequent analyses (the anterior portion of the gluteus medius and the anterior portion of the adductor magnus) based on other practical reasons fully explained in the "Discussion" section.

\section{Ankle Muscle Selection}

All the different ankle muscle sets maintained some subset of the postures remaining after the addition of the hip muscles, but the size and location within the functional stability region of the feasible COM areas varied significantly across muscle sets. The limits of the COM that could be achieved by all the ankle muscles sets are pre-

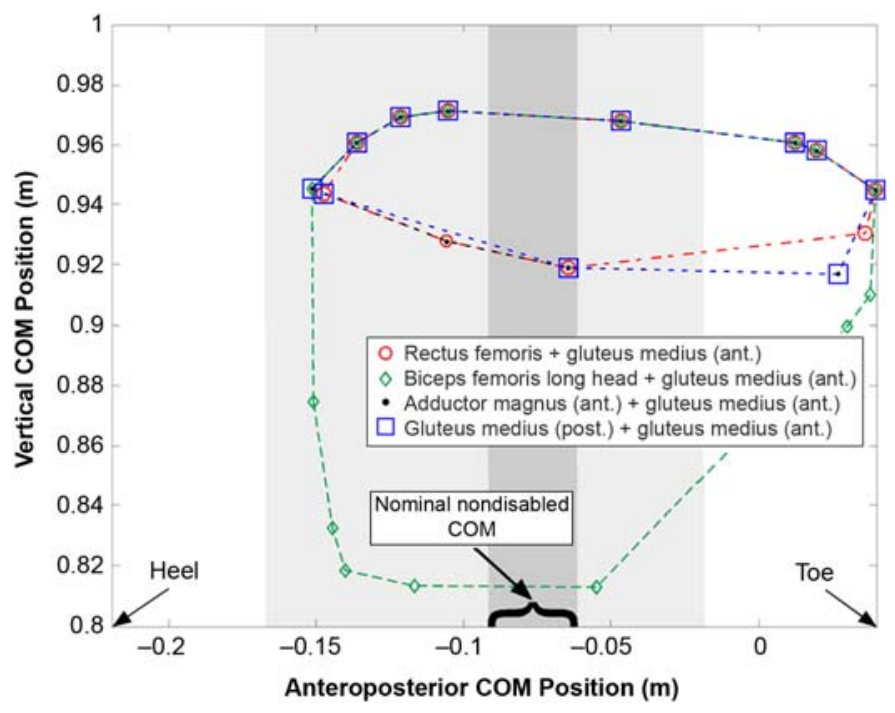

Figure 2.

Limits of COM relative to functional stability region and nominal anteroposterior COM region for each hip muscle combination are shown. Functional stability region is depicted by gray area while anteroposterior region is depicted by darker gray area. Source: Woodhull AM, Maltrud K, Mello BL. Alignment of the human body in standing. Eur J Appl Physiol Occup Physiol. 1985;54(1):109-15 [PMID: 4018044]. ant. = anterior, post. = posterior.

sented in Figure 3(a)-(e). Each graph indicates a different dorsiflexion muscle combination. In each panel, the connected points indicate the boundary of the COM values that could be stabilized by particular sets of added ankle muscles. Different plantar flexion muscle combinations are differentiated by symbols and line types as noted in the legend on the right side of Figure 3(c) and 3(d). The muscle sets that included just the tibialis anterior (Figure 3(a)) or the combination of the tibialis anterior and the peroneus tertius activated equally (Figure 3(c)) covered the largest area of the stability range. The muscle combinations that included stimulation of the peroneal nerve (Figure 3(b)), the superficial peroneal nerve (Figure 3(d)), and proportional activation of the peroneus muscles and the tibialis anterior (Figure 3(e)) spanned a significant range of the BOS limits, but nearly half of that range fell outside of the functional stability region. The plantar flexion combinations that included the soleus (soleus, medial gastrocnemius $=$ lateral gastrocnemius $=$ soleus) were very similar across dorsiflexion combinations. The combinations that included proportional activation of the medial and lateral gastrocnemius covered the largest range of COM values in Figure 3(a) and 3(c) but were the worst plantar flexor combinations in Figure 3(b), 3(d), and 3(e). 


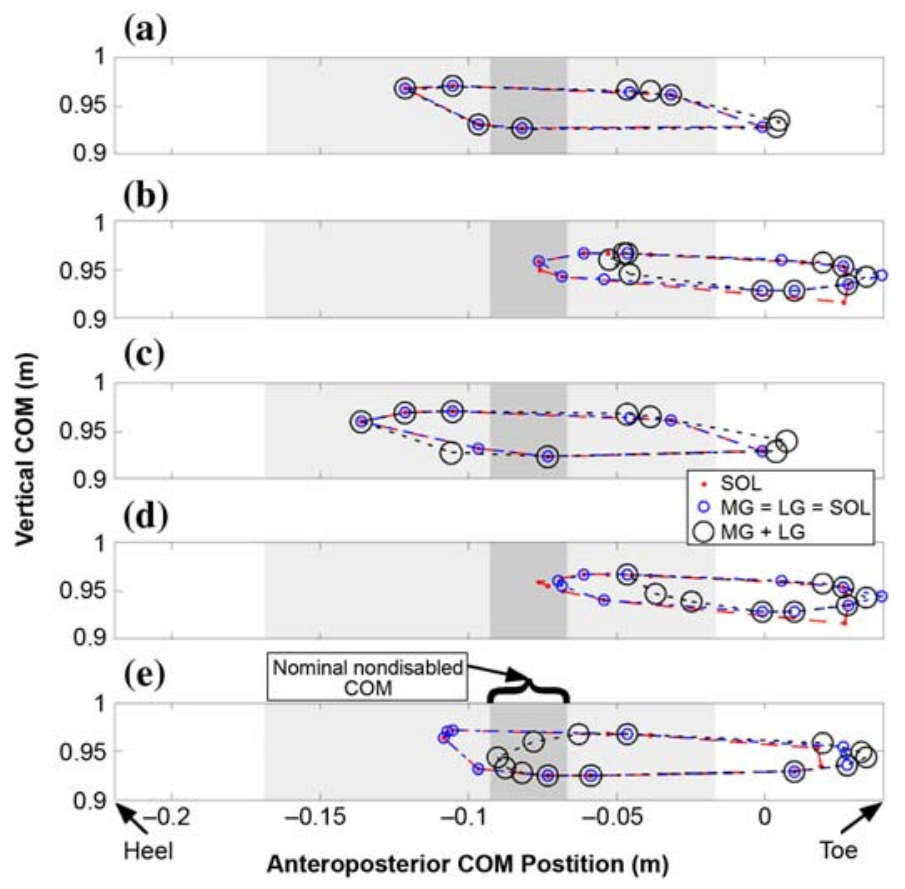

Figure 3.

Center of mass (COM) range for ankle muscle combinations. Limits of range of COM for each ankle muscle combination are shown. (a) TA, (b) $\mathrm{TA}=\mathrm{PB}=\mathrm{PL}=\mathrm{PT}$, (c) $\mathrm{TA}=\mathrm{PT}$, (d) $\mathrm{PB}=\mathrm{PL}$, and (e) $\mathrm{PB}=\mathrm{PL}=$ $\mathrm{PT}=24 \%$ TA. Gray area is limit of functional stability region, with darker gray area being anteroposterior range of nominal able-bodied COM. Source: Woodhull AM, Maltrud K, Mello BL. Alignment of the human body in standing. Eur J Appl Physiol Occup Physiol. 1985;54(1):109-15 [PMID: 4018044]. SOL = soleus, MG = medial gastrocnemius, $\mathrm{LG}=$ lateral gastrocnemius, $\mathrm{PB}=$ peroneus brevis, $\mathrm{PL}$ $=$ peroneus longus, $\mathrm{PT}=$ peroneus tertius, $\mathrm{TA}=$ tibialis anterior .

Figure 4 indicates the percentage of the anteroposterior functional stability region that could be maintained by each muscle set. The percentage is indicated by the vertical axis, and the plantar flexion and dorsiflexion muscle groups are listed along the $x$ - and $y$-axes, respectively. All the plantar flexion combinations combined with the tibialis anterior and the peroneus tertius activated equally covered the largest percentage (78\%) of the functional stability region. All muscle sets that included the superficial peroneal nerve (which innervates the peroneus brevis and longus) or the entire peroneal nerve (which innervates all three peroneal muscles and the tibialis anterior) consistently produced poor results. In addition, activating only the soleus and at the same level as the medial and lateral gastrocnemius produced a larger range across the functional stability region than stimulating the two heads of the gastrocnemius in a proportional manner.

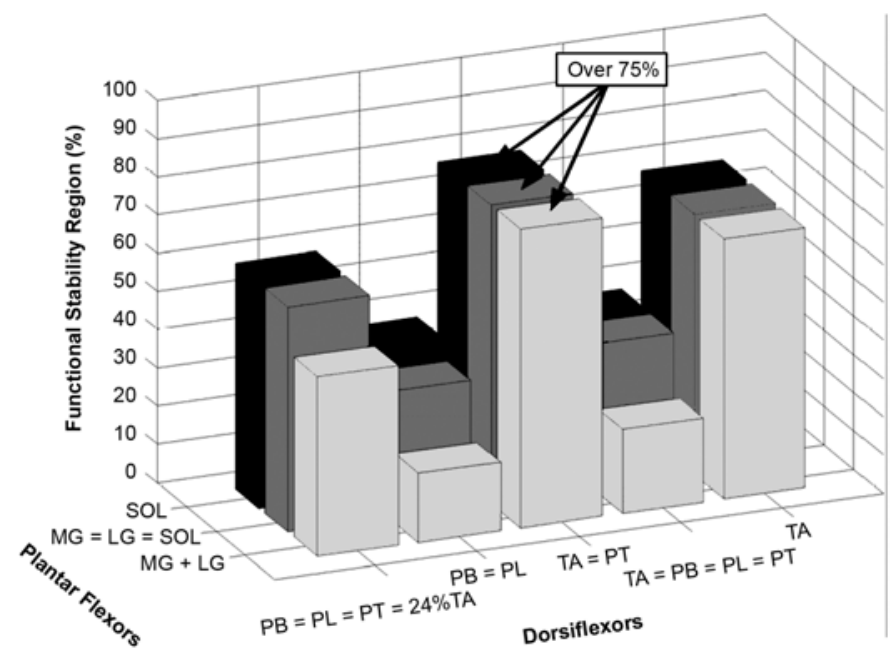

Figure 4.

Ankle combination percentages of functional stability region. Percentage of functional stability region a person with spinal cord injury is to expect with each ankle muscle combination. SOL = soleus, MG = medial gastrocnemius, $\mathrm{LG}=$ lateral gastrocnemius, $\mathrm{PB}=$ peroneus brevis, $\mathrm{PL}=$ peroneus longus, $\mathrm{PT}=$ peroneus tertius, $\mathrm{TA}=$ tibialis anterior.

\section{Mapping Center of Mass Variations via Smooth Joint- Angle Trajectories}

COM joint-angle surfaces were fitted for postures feasible with all muscle combinations that could achieve equilibrium over at least 75 percent of the functional stability region. Figure 5 illustrates typical mapping surfaces that relate the anteroposterior and vertical components of the COM to each of the four joint angles examined. The postures that were feasible with the ankle muscle set consisting of the tibialis anterior, the peroneus tertius (mimicking deep peroneal nerve stimulation), and the soleus were used for this particular plot.

Once we obtained the surfaces, we then used them to define a new set of postures and used the muscle activation optimization procedure to determine the final set of muscles that would maintain those postures. Figure 6 presents the results of these computations for three different muscle combinations. The muscles involved in each result are noted in the caption. The original data set, previously presented in Figure 3, spans the area enclosed by the polygon, and the triangles, circles, and dots represent the position of the COM of 100 postures extracted from the fitted surfaces. The triangles indicate the COM of postures that required more force than the muscle set could provide. The circles represent the positions of the COM of postures that required 50 to 100 percent muscle activation, indicating 
(a)

(b)

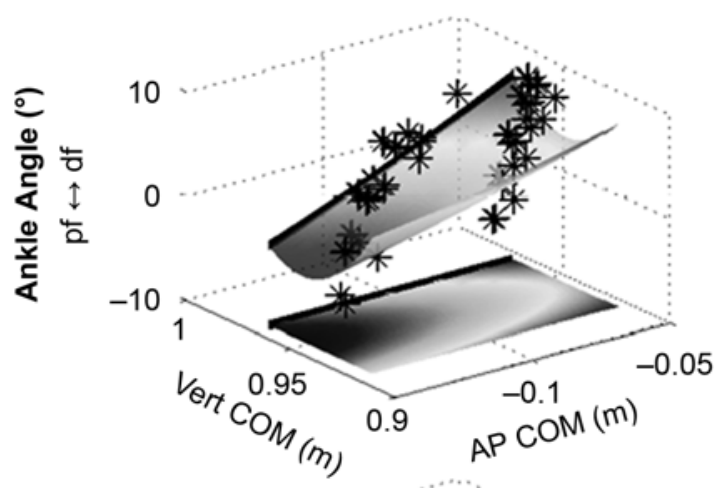

(c)

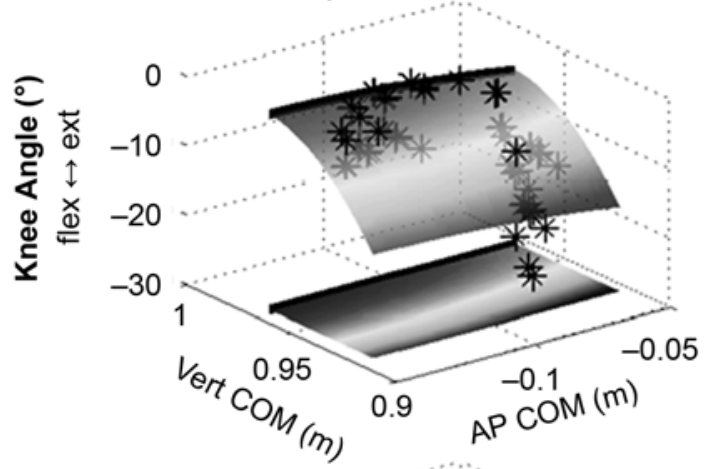

(d)
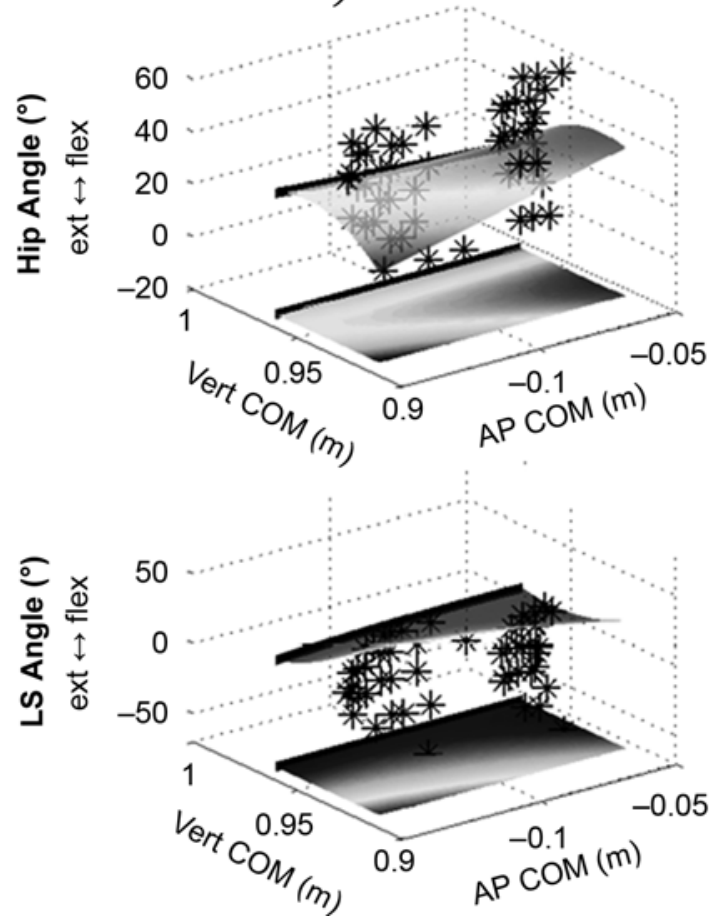

Figure 5.

Vertical (vert) and anteroposterior (AP) center of mass (COM) mapping surfaces. Four plots represent joint angle versus $x$ - and $y$-values of COM for each sagittal plane degree of freedom: (a) ankle, (b) knee, (c) hip, and (d) lumbosacral (LS) angles. Curves were fit to points indicated by stars. Black line on back side of each plot indicates angles for a smooth posture transition in AP direction for most upright postures. $\mathrm{pf}=$ plantar flexion, $\mathrm{df}=$ dorsiflexion, flex = flexion, ext $=$ extension. (a)

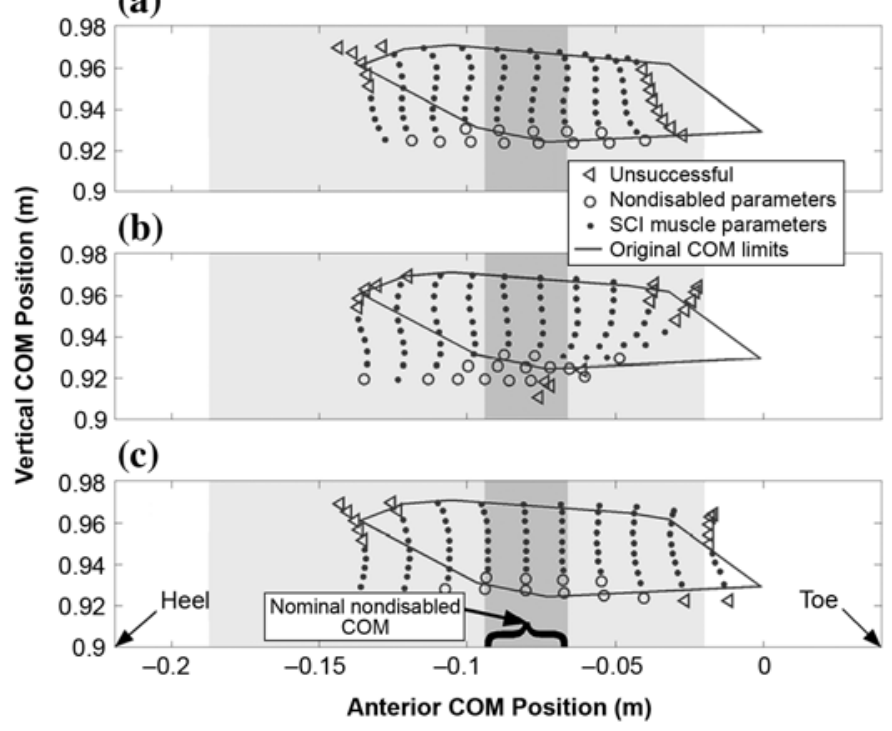

Figure 6.

Smooth center of mass (COM) transitions. COM positions following posture smoothing algorithm for three best ankle combinations are presented: (a) SOL, TA = PT; TA = PT; (b) MG = LG = SOL, TA = PT; and (c) MG + LG, TA = PT. Positions expected to be maintained by a standing-system user are marked with solid black dots. Circles represent nondisabled parameters of postures in which additional muscle strength is required, and triangles correspond to COM positions that muscle set unsuccessfully maintained. SOL = soleus, $\mathrm{TA}=$ tibialis anterior, $\mathrm{MG}=$ medial gastrocnemius, LS = lateral gastrocnemius.

postures that could only be maintained with nondisabled muscle forces. The dots represent the postures requiring muscle activations less than 50 percent and therefore indicate the postures a person with an SCI should be able to maintain. Note that as the COM moves downward, an increased level in muscle activation is required as noted by the circles in the lower section of Figure 6(a) and 6(c). In addition, nearly every posture represented by the COM positions within the polygon was achievable for persons with SCI, along with a significant number of postures with COM values beyond the boundary of the polygons.

\section{Required Muscle Activations}

Figure 7 presents the average muscle activation levels for the ankle-muscle combinations with the largest functional stability region range, also indicating the maximum activations (asterisks) and standard deviations (error bars) across the various postures. The ankle muscles involved in each computation are shown (Figure 7(a) to 7(c)) with the plantar flexion group shown first and the dorsiflexion group second. Since multiple dorsiflexor 


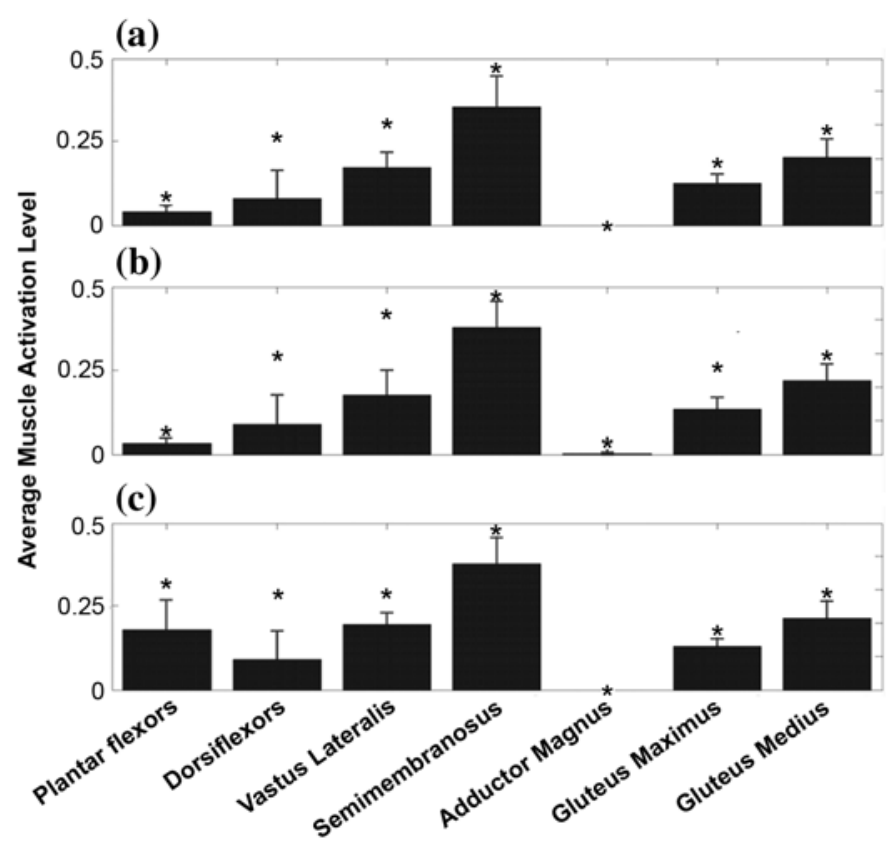

Figure 7.

Average muscle activations required during posture switching. Average and maximum activation levels ${ }^{*}$ of each muscle involved in posture smoothing algorithm are shown. Error bars indicate standard deviation across postures. (a) SOL, TA = PT; (b) $\mathrm{MG}=\mathrm{LG}=\mathrm{SOL}$, TA = PA; (c) $\mathrm{MG}=\mathrm{LG}, \mathrm{TA}=\mathrm{PT}$. SOL = soleus, $\mathrm{TA}=$ tibialis anterior, $\mathrm{PT}=$ peroneas tertius, $\mathrm{MG}=$ medial gastrocnemius, $\mathrm{PG}=$ lateral gastrocnemius.

muscles and multiple plantar flexor muscles were used, the muscle with the highest activation is illustrated. All the muscles except the semimembranosus required only modest activation levels for the postures tested, with averages less than 25 percent of maximum. The semimembranosus was consistently activated at the highest level. The adductor magnus did not have a significant role in the sagittal plane postures examined, but it is expected to be essential for coronal plane movements. Only the plantar flexor muscle activations had noticeable differences in average activation values for the four different muscle combinations. The combinations including the soleus and the soleus and both the gastrocnemius heads were much less active than the combination including the proportional activations of the medial and lateral gastrocnemius.

\section{DISCUSSION}

We used a human musculoskeletal model to evaluate the likely effects of adding eight additional muscles to the current CWRU/VAMC standing system. Including these additional muscles should reduce users' reliance on the upper limbs for support while standing and will thus allow them, at a minimum, to release one hand to perform functional activities. Overall, we found that a number of different muscle combinations could support hands-free standing, indicating a fair degree of flexibility in specifying the muscle set for individual subjects. These results indicate that a 16-channel FES system should be capable of allowing users to shift postures over a significant percentage of the forward-backward range used by nondisabled individuals during typical activities.

\section{Hip Muscle Selection}

Even though several hip muscle combinations resulted in similar performance, the combination of the anterior portion of the adductor magnus and the anterior portion of the gluteus medius was selected for two reasons. First, the uniarticular adductor magnus and gluteus medius should be easier to control than the rectus femoris and the long head of the biceps femoris, which are both biarticular muscles. Second, the combination provides the potential to allow coronal plane posture shifts because of its adduction actions, while the other candidate muscles (biceps femoris long head, posterior portion of the gluteus medius) largely replicate the actions of other muscles already used in the 8-channel system.

\section{Ankle Muscle Selection}

We also evaluated several different combinations of stimulation at the ankle (two channels bilaterally). Most of the tested ankle muscle combinations supported the body over more than 50 percent of the functional stability region, and three of these combinations supported the body over more than 75 percent of the functional stability region. The simplest complete ankle muscle set only requires the soleus and the tibialis anterior, and the medial and lateral gastrocnemii could be added for additional plantar flexion. If the ankle was consistently being inverted, the peroneus muscles could be added. Thus, some flexibility in the choice of ankle muscles to include in the expanded FES standing system exists. Adding the medial and lateral gastrocnemii to the soleus showed no improvement over activating the soleus alone, suggesting that the knee flexion created by activating the gastrocnemii might not warrant recruiting them along with the soleus. Across all combinations, the activation levels required of the ankle plantar flexor and dorsiflexor muscles were low, indicating a reserve capacity that should allow users to 
perform functional tasks while standing and to compensate for muscle weakness or muscle fatigue.

\section{Center of Mass Mapping Performance}

Our primary motivation for this study was to propose additional muscles that would allow FES standingsystem users to adjust their own posture while standing. For example, the user could send signals to the neuroprosthesis to adjust anterior-posterior and lateral shifts via a finger-worn joystick. Indeed, we based our evaluation of potential muscle sets on each set being able to provide smooth changes in the location of the COM via smooth changes in each of the four joint angles. A given COM can be achieved by many different combinations of joint angles. We simultaneously fitted polynomial surfaces through the points in Figure 5(a) to (d) to define one way of providing smooth joint-angle variations with the commanded COM varied throughout its feasible range and avoiding large changes or even reversals in commanded joint angles as the COM varied slightly. Other mappings are certainly possible and could be achieved if one were to fit alternative functions through the clouds of possible solutions presented in Figure 5 or by optimizing other features, for example, constraining the knee to remain locked in full extension.

\section{Effect of Model Assumptions}

The model we used in this study accurately represents the human musculoskeletal system, but the overall accuracy of the results we obtained depends on the numerous assumptions made to emulate an individual with paraplegia standing via a 16-channel FES system. First, obtaining the needed muscle forces from paralyzed and atrophied muscles in some individuals may not be possible. In such cases, an interdisciplinary team of professionals would need to evaluate the maximum stimulated strength of the muscles in a particular individual to select the optimal muscle set, which could include synergistic muscles to guarantee sagittal plane function rather than attempting to add additional coronal plane function. Second, we did not simulate every possible muscle combination, so an alternative muscle set could have possibly given a more optimal result. This seems unlikely, however, because our muscle selection criteria included all the primary movers at the critical $d f$ that are not controlled by the current 8-channel system and because our choices were guided by years of clinical experience [67,19-20,25]. However, some major muscles, including the posterior portion of the adductor magnus, were excluded from the hip muscle evaluation because they did not generate the largest moment in one of the six directions of hip motion. Third, we did not consider additional muscles of the trunk, for example the abdominal muscles or additional extensor muscles. Although such additional muscles may prove useful in future systems, we believe that the ankle and hip remain the most critical joints for successfully controlling standing via FES, considering nondisabled persons maintain balance by actively changing the hip and ankle angles [26]. The ankle, by virtue of its distal location, has the largest impact on the ability to shift the COM. Hip extension has proven to be a limiting factor in some current 8-channel system users and abduction-adduction actions of the existing hip extensor muscles have not been actively balanced.

\section{Standing System Implications}

The goal of this study was to guide the muscle selection process for the next generation standing system and to determine if a subset of muscles could theoretically support a person without natural lower-limb muscle control. The goal was achieved for several different combinations of muscles, so even in the case of a particularly weak muscle, an alternative muscle set could be selected. The results obtained here should also benefit the development of strategies for closed-loop control of standing balance.

The next step in the development of the 16-channel FES standing system is to implement it in human volunteers. Such a system would provide significant functional benefits to the users and would allow us to rigorously validate the model-based approach presented here. In the validation process, we would use the new 16-channel stimulator for surface stimulation of the muscles of SCI volunteers in a controlled environment, and we would stimulate different muscle combinations to provide cocontraction for standing. Thereafter, the volunteers would be asked to adjust the stimulation levels of the selected muscles and the ground reaction forces at the 2 feet captured so the effect of selected muscle combination on posture shifting could be determined.

\section{CONCLUSION}

We have carried out extensive computer simulation studies to determine an optimal set of the lower-limb muscles that could be stimulated electrically to allow 
individuals with SCIs using the 16-channel FES system to smoothly shift their postures to minimize fatigue. The muscles selected would augment the current set of eight muscles (four bilaterally) used to produce erect standing from the seated position. By carefully analyzing different combinations of muscles, we concluded that adding gluteus medius and adductor magnus at the hip, soleus, and tibialis anterior at the ankle (all bilaterally) was sufficient to shift postures over 75 percent of the forward-backward range that nondisabled individuals typically use. This information has potential clinical application in the rehabilitation of SCI individuals using new 16-channel FES systems.

\section{ACKNOWLEDGMENTS}

Mr. Heilman is now with Temple University, Philadelphia, Pennsylvania.

This material was based on work supported by the VA Rehabilitation Research and Development merit review, grant B3025.

The authors have declared that no competing interests exist.

\section{REFERENCES}

1. Stover SL, DeLisa JA, Whiteneck GG. Spinal cord injury: clinical outcomes from the model systems. Gaithersburg (MD): Aspen Publishers; 1995. p. 21-51.

2. Phillips B, Zhao H. Predictors of assistive technology abandonment. Assist Technol. 1993;5(1):36-45. [PMID: 10171664]

3. Brindley GS, Polkey CE, Rushton DN. Electrical splinting of the knee in paraplegia. Paraplegia. 1979;16(4):428-37. [PMID: 311910]

4. Bajd T, Kralj A, Sega J, Turk R, Benko H, Strojnik P. Use of a two-channel functional electrical stimulator to stand paraplegic patients. Phys Ther. 1981;61(4):526-27. [PMID: 208637]

5. Krajl A, Bajd T, Turk R, Benko H. Posture switching for prolonging functional electrical stimulation standing in paraplegic patients. Paraplegia. 1986;24(4):221-30. [PMID: 3489917$]$

6. Triolo RJ, Bieri C, Uhlir JP, Kobetic R, Scheiner A, Marsolais EB. Implanted Functional Neuromuscular Stimulation systems for individuals with cervical spinal cord injuries: clinical case reports. Arch Phys Med Rehabil. 1996; 77(11):1119-28. [PMID: 8931521]

7. Davis JA Jr, Triolo RJ, Uhlir JP, Bhadra N, Lissy DA, Nandurkar S, Marsolais EB. Surgical technique for install- ing an eight-channel neuroprosthesis for standing. Clin Orthop Relat Res. 2001;(385):237-52. [PMID: 11302320$]$

8. Davis JA Jr, Triolo RJ, Uhlir JP, Bieri C, Rohde L, Lissy DA, Kukke S. Preliminary performance of a surgically implanted neuroprosthesis for standing and transferswhere do we stand? J Rehabil Res Dev. 2001;38(6):609-17. [PMID: 11767968]

9. Betz R, Boden B, Triolo RJ, Mesgarzadeh M, Gardner E, Fife R. Effects of functional electrical stimulation on the joints of adolescents with spinal cord injury. Paraplegia. 1996;34(3):127-36. [PMID: 8668353]

10. Bogie KM, Triolo RJ. Effects of regular use of neuromuscular electrical stimulation on tissue health. J Rehabil Res Dev. 2003;40(6):469-75. [PMID: 15077659]

11. Hunt KJ, Gollee H, Jaime RP. Control of paraplegic ankle joint stiffness using FES while standing. Med Eng Phys. 2001;23(8):541-55. [PMID: 11719077]

12. Audu ML, Kirsch RF, Triolo RJ. A computational technique for determining the ground reaction forces in human bipedal stance. J Appl Biomech. 2003;19(4):361-71.

13. Marsolais EB, Kobetic R. Implantation techniques and experience with percutaneous intramuscular electrodes in the lower extremities. J Rehabil Res Dev. 1986;23(3):1-8. [PMID: 490566]

14. Zajac FE. Muscle and tendon: properties, models, scaling, and application to biomechanics and motor control. Crit Rev Biomed Eng. 1989;17(4):359-411. [PMID: 2676342]

15. Brand RA, Pedersen DR, Friederich JA. The sensitivity of muscle force predictions to changes in physiologic crosssectional area. J Biomech. 1986;19(8):589-96.

[PMID: 3771581]

16. Wickiewicz TL, Roy RR, Powell PL, Edgerton VR. Muscle architecture of the human lower limb. Clin Orthop Relat Res. 1983;(179):275-83. [PMID: 6617027]

17. Friederich JA, Brand RA. Muscle fiber architecture in the human lower limb. J Biomech. 1990;23(1):91-95. [PMID: 2307696]

18. Triolo RJ, Liu MQ, Kobetic R, Uhlir JP. Selectivity of intramuscular stimulating electrodes in the lower limbs. J Rehabil Res Dev. 2001;38(5):533-44. [PMID: 11732831]

19. Uhlir JP, Triolo RJ, Kobetic R. The use of selective electrical stimulation of the quadriceps to improve standing function in paraplegia. IEEE Trans Rehabil Eng. 2000;8(4): 514-22. [PMID: 11204043]

20. Marsolais EB, Kobetic R. Functional electrical stimulation for walking in paraplegia. J Bone Joint Surg Am. 1987; 69(5):728-33. [PMID: 3496340]

21. Smith SS, Mayer TG, Gatchel RJ, Becker TJ. Quantification of lumbar function. Part 1: Isometric and multispeed isokinetic trunk strength measures in sagittal and axial planes in normal subjects. Spine. 1985;10(8):757-64. [PMID: 4081883] 
22. Amankwah K, Triolo RJ, Kirsch RF. Effects of spinal cord injury on lower-limb passive joint moments revealed through a nonlinear viscoelastic model. J Rehabil Res Dev. 2004;41(1):15-32. [PMID: 15273894]

23. Crowninshield RD, Brand RA. A physiologically based criterion of muscle force prediction in locomotion. J Biomech. 1981;14(11):793-801. [PMID: 7334039]

24. Holbein MA, Redfern MS. Functional stability limits while holding loads in various positions. Int J Ind Ergon. 1997; 19(5):387-95. [PMID: 11540602]

25. Woodhull AM, Maltrud K, Mello BL. Alignment of the human body in standing. Eur J Appl Physiol Occup Physiol. 1985;54(1):109-15. [PMID: 4018044$]$
26. Kobetic R, Triolo RJ, Marsolais EB. Muscle selection and walking performance of multichannel FES systems for ambulation in paraplegia. IEEE Trans Rehabil Eng. 1997;5(1):23-29. [PMID: 9086382]

27. Horak FB, Nashner LM. Central programming of postural movements: adaptation to altered support-surface configurations. J Neurophysiol. 1986;55(6):1369-81. [PMID: 3734861]

Submitted for publication April 21, 2005. Accepted in revised form September 26, 2005. 